\title{
Duality Between Smooth Min- and Max-Entropies
}

\author{
Marco Tomamichel,, , 田 Roger Colbeck, ${ }^{1,2, \text {, }}$ and Renato Renner ${ }^{1, \text { f }}$ \\ ${ }^{1}$ Institute for Theoretical Physics, ETH Zurich, 8093 Zurich, Switzerland. \\ ${ }^{2}$ Institute of Theoretical Computer Science, ETH Zurich, 8092 Zurich, Switzerland.
}

(Dated: October 20, 2009)

\begin{abstract}
In classical and quantum information theory, operational quantities such as the amount of randomness that can be extracted from a given source or the amount of space needed to store given data are normally characterized by one of two entropy measures, called smooth min-entropy and smooth max-entropy, respectively. While both entropies are equal to the von Neumann entropy in certain special cases (e.g., asymptotically, for many independent repetitions of the given data), their values can differ arbitrarily in the general case.

In this work, a recently discovered duality relation between (non-smooth) min- and max-entropies is extended to the smooth case. More precisely, it is shown that the smooth min-entropy of a system A conditioned on a system B equals the negative of the smooth max-entropy of A conditioned on a purifying system $\mathrm{C}$. This result immediately implies that certain operational quantities (such as the amount of compression and the amount of randomness that can be extracted from given data) are related. Such relations may, for example, have applications in cryptographic security proofs.
\end{abstract}

\section{INTRODUCTION}

Entropies are used to quantitatively characterize problems in quantum information processing and quantum cryptography. In the case of many independent and identical instances of a task (i.i.d. limit), the von Neumann entropy is the relevant measure. In order to go beyond this restriction, the smooth min- and max-entropies have been introduced. The smooth min-entropy was introduced in order to characterize randomness extraction. It corresponds to the length of uniform random string that can be generated from a partially unifrom one [1, 2]. The smooth max-entropy, on the other hand, was introduced to characterize information reconciliation. It gives the amount of communication required between two parties in order that they can generate a perfectly correlated string from a partially correlated one 3]. Since their initial uses, these entropies have found applications in many tasks (see for example [4, [5]) and have been shown to converge to the von Neumann entropy in the i.i.d. limit [2, 6].

The smooth entropies can be defined as optimizations of the relevant non-smooth quantities - the (nonsmooth) min- and max-entropies - over a set of nearby states. 'Nearby' is specified via a smoothing parameter, the maximum distance from the original state in an appropriate metric (for precise definitions, see below). Often, the smooth entropy is the correct measure when one accounts for a small error tolerance, whereas the nonsmooth entropy characterizes the zero error case. In the case of privacy amplification, for example, ideally one wants a protocol in which two parties, Alice (A) and Bob (B), use a shared string about which an eavesdropper (E) has partial information and form a secret key about which E knows nothing. Unfortunately, such a stringent requirement is usually unattainable. Instead, one tolerates a small probability that the eavesdropper learns something about the key in order to achieve a significant key length. In this case, the smooth min-entropy of A given $\mathrm{E}$ characterizes the length of the key, with the smoothing parameter dependent on the tolerable error [1].

It has recently been discovered that the min- and maxentropies are related [7]. They are dual to one another in the sense that for a pure state $\rho_{\mathrm{ABC}}$ on a tri-partite Hilbert space $\mathcal{H}_{\mathrm{A}} \otimes \mathcal{H}_{\mathrm{B}} \otimes \mathcal{H}_{\mathrm{C}}$, the conditional min-entropy of $\mathrm{A}$ given $\mathrm{B}$ is the negative of the conditional maxentropy of A given C, i.e. $H_{\min }(\mathrm{A} \mid \mathrm{B})_{\rho}=-H_{\max }(\mathrm{A} \mid \mathrm{C})_{\rho}$. In this work, we extend the duality relation to the smooth min- and max-entropies. In order to do this, a new method of smoothing is required: We propose measuring the closeness of states used in the definition of the smooth entropies in terms of a quantity which we call the purified distance. This forms a metric on the set of sub-normalized states (positive semi-definite operators with trace at most 1). When defined in this way, the smooth min- and max-entropies satisfy various natural properties such as invariance under local isometries and the data processing inequality (that quantum operations cannot decrease entropy). The duality not only simplifies many derivations ${ }^{1}$, but it provides a connection between seemingly unrelated problems. In particular, this means that randomness extraction and information reconciliation can be characterized by the same entropy.

In the context of quantum key distribution, the new relation has the following application. In order to generate a secure key, Alice and Bob need to bound the smooth

\footnotetext{
${ }^{1}$ Various relations for the min-entropy hold automatically for the max-entropy via the duality, e.g. the fact that conditioning on an additional system always reduces entropy (cf. Theorem 18).
}

marcoto@phys.ethz.ch

trenner@phys.ethz.ch 
min-entropy of A conditioned on E. Our result provides a way for them to generate this bound without access to the eavesdropper's systems. In the worst case, the eavesdropper holds a purification of the state of A and B. (The data processing inequality (Theorem [18) implies that if she does not, her information about the key is strictly smaller.) Using the duality relation, Alice and Bob obtain the desired bound on the smooth min-entropy by estimating the smooth max-entropy of A given $\mathrm{B}$.

There is an alternative method for going beyond i.i.d. in information theory, known as the information spectrum method [8, 9, 10]. Like for smooth entropies, there are two principal quantities: the inf-spectral entropy rate which is related to the smooth min-entropy and the supspectral entropy rate which is related to the smooth maxentropy [11]. The results of this paper imply that a similar duality relation holds for the spectral entropy rates.

The remainder of this paper is organized as follows. In Section II we introduce the purified distance and prove that it is a metric on sub-normalized states. In Section III we use this metric to define a ball of states around a particular state. This ball is then used to define the smooth conditional min- and max-entropies in Section [V] and to prove that they satisfy data processing inequalities in Section $\nabla$

\section{METRICS ON THE SET OF SUB-NORMALIZED STATES}

Let $\mathcal{H}$ be a finite-dimensional Hilbert space. We use $\mathcal{L}(\mathcal{H})$ and $\mathcal{P}(\mathcal{H})$ to denote the set of linear operators on $\mathcal{H}$ and the set of positive semi-definite operators on $\mathcal{H}$, respectively. We define the set of normalized quantum states by $\mathcal{S}_{=}(\mathcal{H}):=\{\rho \in \mathcal{P}(\mathcal{H}): \operatorname{tr} \rho=1\}$ and the set of sub-normalized states by $\mathcal{S}_{\leq}(\mathcal{H}):=\{\rho \in \mathcal{P}(\mathcal{H}): 0<$ $\operatorname{tr} \rho \leq 1\}$. Note that $\mathcal{L}(\mathcal{H}) \supset \overline{\mathcal{P}}(\mathcal{H}) \supset \mathcal{S}_{\leq}(\mathcal{H}) \supset \mathcal{S}_{=}(\mathcal{H})$ Given a pure state $|\phi\rangle \in \mathcal{H}$, we use $\phi=\overline{\mid} \phi\rangle\langle\phi|$ to denote the corresponding projector in $\mathcal{P}(\mathcal{H})$.

We start by introducing a generalization of the trace distance:

Definition 1. For $\rho, \tau \in \mathcal{P}(\mathcal{H})$, we define the generalized trace distance between $\rho$ and $\tau$ as

$$
\bar{D}(\rho, \tau):=\max \left\{\operatorname{tr}\{\rho-\tau\}_{+}, \operatorname{tr}\{\tau-\rho\}_{+}\right\},
$$

where $\{X\}_{+}$denotes the projection of $X$ onto its positive eigenspace.

In the case of normalized states, we have $\operatorname{tr}\{\rho-\tau\}_{+}=$ $\operatorname{tr}\{\tau-\rho\}+$ and recover the usual trace distance $D(\rho, \tau):=$ $\operatorname{tr}\{\rho-\tau\}_{+}$. The generalized trace distance can alternatively be expressed in terms of the Schatten 1-norm $\|X\|_{1}=\operatorname{tr}|X|=\operatorname{tr} \sqrt{X^{\dagger} X}$ as

$$
\bar{D}(\rho, \tau)=\frac{1}{2}\|\rho-\tau\|_{1}+\frac{1}{2}|\operatorname{tr} \rho-\operatorname{tr} \tau|
$$

and it is easy to verify that it is a metric on $\mathcal{L}(\mathcal{H})$. The trace distance has a physical interpretation as the distinguishing advantage between two normalized states. In other words, the probability $p_{\text {dist }}(\rho, \tau)$ of correctly guessing which of two equiprobable states $\rho$ and $\tau$ is provided is upper bounded by 12 ]

$$
p_{\text {dist }}(\rho, \tau) \leq \frac{1}{2}(1+D(\rho, \tau)) .
$$

Various quantities derived from the fidelity $F(\rho, \tau)=$ $\|\sqrt{\rho} \sqrt{\tau}\|_{1}$ are used in the literature to quantify the distance between normalized states. Its generalization to sub-normalized states satisfies $0 \leq F(\rho, \tau) \leq$ $\sqrt{\operatorname{tr} \rho} \sqrt{\operatorname{tr} \tau}$ and is monotonically increasing under trace preserving completely positive maps (TP-CPMs), i.e. $F(\mathcal{E}(\rho), \mathcal{E}(\tau)) \geq F(\rho, \tau)$ for any TP-CPM $\mathcal{E}$ (cf. [12], Theorem 9.6). Moreover, we will often use Uhlmann's theorem [13] which states that, for any purification $\varphi$ of $\rho$, there exists a purification $\vartheta$ of $\tau$ such that $F(\rho, \tau)=$ $F(\varphi, \vartheta)=|\langle\varphi \mid \vartheta\rangle|$. The fidelity is also symmetric in its arguments, i.e. $F(\rho, \tau)=F(\tau, \rho)$.

For our argument, we need an alternative generalization of the fidelity to sub-normalized states. The generalization is motivated by the observation that subnormalized states can be thought of as normalized states on a larger space projected onto a subspace. We write $\overline{\mathcal{H}} \supseteq \mathcal{H}$ if a Hilbert space $\mathcal{H}$ is embedded in another Hilbert space $\overline{\mathcal{H}}$ and denote the projector onto $\mathcal{H}$ by $\Pi$.

Definition 2. For $\rho, \tau \in \mathcal{S}_{\leq}(\mathcal{H})$, we define the generalized fidelity between $\rho$ and $\tau$ as

$$
\bar{F}(\rho, \tau):=\sup _{\overline{\mathcal{H}} \supseteq \mathcal{H}} \sup _{\substack{\bar{\rho}, \bar{\tau} \in \mathcal{S}_{=}(\overline{\mathcal{H}}) \\ \rho=\Pi \bar{\rho} \Pi, \tau=\Pi \bar{\tau} \Pi}} F(\bar{\rho}, \bar{\tau}) .
$$

Note that $\bar{F}$ reduces to $F$ when at least one state is normalized. This can be seen from the following alternative expression for $\bar{F}$ :

Lemma 3. Let $\rho, \tau \in \mathcal{S}_{\leq}(\mathcal{H})$. Then,

$$
\bar{F}(\rho, \tau)=F(\hat{\rho}, \hat{\tau})=F(\rho, \tau)+\sqrt{(1-\operatorname{tr} \rho)(1-\operatorname{tr} \tau)},
$$

where $\hat{\rho}:=\rho \oplus(1-\operatorname{tr} \rho)$ and $\hat{\tau}:=\tau \oplus(1-\operatorname{tr} \tau)$.

Proof. Let $\overline{\mathcal{H}}, \bar{\rho}$ and $\bar{\tau}$ be any combination of Hilbert space and states that are candidates for the maximization in (2). Let $\mathcal{E}: \overline{\mathcal{H}} \rightarrow \overline{\mathcal{H}}$ be the pinching $\mathcal{E}: \bar{\rho} \mapsto$ $\Pi \bar{\rho} \Pi+\Pi_{\perp} \bar{\rho} \Pi_{\perp}$, where $\Pi$ is the projector onto $\mathcal{H}$ and $\Pi_{\perp}=\mathbb{1}_{\overline{\mathcal{H}}}-\Pi$ its orthogonal complement. Hence,

$$
\begin{aligned}
F(\bar{\rho}, \bar{\tau}) & \leq F(\mathcal{E}(\bar{\rho}), \mathcal{E}(\bar{\tau})) \\
& =F(\rho, \tau)+F\left(\Pi_{\perp} \bar{\rho} \Pi_{\perp}, \Pi_{\perp} \bar{\tau} \Pi_{\perp}\right) \\
& \leq F(\rho, \tau)+\sqrt{(1-\operatorname{tr} \rho)(1-\operatorname{tr} \tau)} .
\end{aligned}
$$

It is easy to verify that the upper bound is achieved by $\hat{\mathcal{H}}=\mathcal{H} \oplus \mathbb{C}, \hat{\rho}$ and $\hat{\tau}$.

We define a metric based on the fidelity, analogously to the one proposed in $[14,15]^{2}$ :

\footnotetext{
2 The quantity $C(\rho, \tau)=\sqrt{1-F^{2}(\rho, \tau)}$ is introduced in [14],
} 
Definition 4. For $\rho, \tau \in \mathcal{S}_{\leq}(\mathcal{H})$, we define the purified distance between $\rho$ and $\tau$ as

$$
P(\rho, \tau):=\sqrt{1-\bar{F}(\rho, \tau)^{2}}
$$

The name is motivated by the fact that, for normalized states $\rho, \tau \in \mathcal{S}_{=}(\mathcal{H})$, we can write $P(\rho, \tau)$ as the minimum trace distance between purifications $|\varphi\rangle$ of $\rho$ and $|\vartheta\rangle$ of $\tau$. More precisely, using Uhlmann's theorem [13], we have

$$
\begin{aligned}
P(\rho, \tau) & =\sqrt{1-F(\rho, \tau)^{2}}=\sqrt{1-\max _{\varphi, \vartheta}|\langle\varphi \mid \vartheta\rangle|^{2}} \\
& =\min _{\varphi, \vartheta} \sqrt{1-|\langle\varphi \mid \vartheta\rangle|^{2}}=\min _{\varphi, \vartheta} \bar{D}(\varphi, \vartheta) .
\end{aligned}
$$

Lemma 5. The purified distance $P$ is a metric on $\mathcal{S}_{\leq}(\mathcal{H})$.

Proof. Let $\rho, \tau$ and $\sigma$ be any states in $\mathcal{S}_{\leq}(\mathcal{H})$. The condition $P(\rho, \tau)=0 \Longleftrightarrow \rho=\tau$ can be verified by inspection, and symmetry $P(\rho, \tau)=P(\tau, \rho)$ follows from the symmetry of the fidelity.

It remains to show the triangle inequality $P(\rho, \tau) \leq$ $P(\rho, \sigma)+P(\sigma, \tau)$. Using Lemma 3, the generalized fidelities between $\rho, \tau$ and $\sigma$ can be expressed as fidelities between the corresponding extensions $\hat{\rho}, \hat{\tau}$ and $\hat{\sigma}$. Furthermore, we use Uhlmann's theorem to introduce purifications $|r\rangle$ of $\hat{\rho},|s\rangle$ of $\hat{\sigma}$ and $|t\rangle$ of $\hat{\tau}$ such that $F(\hat{\rho}, \hat{\sigma})=|\langle r \mid s\rangle|, F(\hat{\sigma}, \hat{\tau})=|\langle s \mid t\rangle|$ and $F(\hat{\rho}, \hat{\tau}) \geq|\langle r \mid t\rangle|$. Hence,

$$
\begin{aligned}
P(\rho, \sigma)+P(\sigma, \tau) & =P(r, s)+P(s, t) \\
& =D(r, s)+D(s, t) \\
& \geq D(r, t)=P(r, t) \geq P(\rho, \tau),
\end{aligned}
$$

where we have used the triangle inequality for the trace distance.

The following lemma gives lower and upper bounds to the purified distance in terms of the generalized trace distance.

Lemma 6. Let $\rho, \tau \in \mathcal{S}_{\leq}(\mathcal{H})$. Then

$$
\bar{D}(\rho, \tau) \leq P(\rho, \tau) \leq \sqrt{2 \bar{D}(\rho, \tau)} .
$$

Proof. We express the quantities using the normalized extensions $\hat{\rho}$ and $\hat{\tau}$ of Lemma 3 to get

$P(\rho, \tau)=\sqrt{1-F(\hat{\rho}, \hat{\tau})^{2}} \geq D(\hat{\rho}, \hat{\tau})=\bar{D}(\rho, \tau) \quad$ and

$P(\rho, \tau)^{2}=1-F(\hat{\rho}, \hat{\tau})^{2} \leq 1-(1-D(\hat{\rho}, \hat{\tau}))^{2} \leq 2 \bar{D}(\rho, \tau)$,

where we have made use of $1-F(\hat{\rho}, \hat{\tau}) \leq D(\hat{\rho}, \hat{\tau}) \leq$ $\sqrt{1-F(\hat{\rho}, \hat{\tau})^{2}}$ (see e.g. [12], Section 9.2.3).

where they also show that it is a metric on $\mathcal{S}_{=}(\mathcal{H})$. In [15] the quantity is called sine distance and some of its properties are explored.
A useful property of the purified distance is that it does not increase under simultaneous application of a quantum operation on both states. We consider the class of trace non-increasing CPMs, which includes projections.

Lemma 7. Let $\rho, \tau \in \mathcal{S}_{\leq}(\mathcal{H})$ and $\mathcal{E}$ be a trace nonincreasing CPM. Then, $P(\rho, \tau) \geq P(\mathcal{E}(\rho), \mathcal{E}(\tau))$.

Proof. Note that a trace non-increasing $\mathrm{CPM} \mathcal{E}$ : $\mathcal{P}(\mathcal{H}) \rightarrow \mathcal{P}\left(\mathcal{H}^{\prime}\right)$ can be decomposed into an isometry $U$ : $\mathcal{H} \rightarrow \mathcal{H}^{\prime} \otimes \mathcal{H}^{\prime \prime}$ followed by a projection $\Pi \in \mathcal{P}\left(\mathcal{H}^{\prime} \otimes \mathcal{H}^{\prime \prime}\right)$ and a partial trace over $\mathcal{H}^{\prime \prime}$ (see, e.g. [12], Section 8.2). The isometry and the partial trace are TP-CPMs and, hence, it suffices to show that $\bar{F}(\rho, \tau) \leq \bar{F}(\mathcal{E}(\rho), \mathcal{E}(\tau))$ for TP-CPMs and projections.

First, let $\mathcal{E}$ be trace preserving. Using Lemma 3 and the monotonicity under TP-CPMs of the fidelity, we see that

$$
\begin{aligned}
\bar{F}(\rho, \tau) & =F(\rho, \tau)+\sqrt{(1-\operatorname{tr} \rho)(1-\operatorname{tr} \tau)} \\
& \leq F(\mathcal{E}(\rho), \mathcal{E}(\tau))+\sqrt{(1-\operatorname{tr} \rho)(1-\operatorname{tr} \tau)} \\
& =\bar{F}(\mathcal{E}(\rho), \mathcal{E}(\tau)) .
\end{aligned}
$$

Next, consider a projection $\Pi \in \mathcal{P}(\mathcal{H})$ and the $\mathrm{CPM}$ $\mathcal{E}: \rho \mapsto \Pi \rho \Pi$. Following Definition 2 , we write $\bar{F}(\rho, \tau)=$ sup $F(\bar{\rho}, \bar{\tau})$, where the supremum is taken over all extensions $\{\overline{\mathcal{H}}, \bar{\rho}, \bar{\tau}\}$ of $\{\mathcal{H}, \rho, \tau\}$. Since all extensions of $\{\mathcal{H}, \rho, \tau\}$ are also extensions of $\{\operatorname{supp}\{\Pi\}, \Pi \rho \Pi, \Pi \tau \Pi\}$, we find $\bar{F}(\Pi \rho \Pi, \Pi \tau \Pi) \geq \bar{F}(\rho, \tau)$.

The main advantage of the purified distance over the trace distance is that we can always find extensions and purifications without increasing the distance.

Lemma 8. Let $\rho, \tau \in \mathcal{S}_{\leq}(\mathcal{H}), \mathcal{H}^{\prime} \cong \mathcal{H}$ and $\varphi \in \mathcal{H} \otimes \mathcal{H}^{\prime}$ be a purification of $\rho$. Then, there exists a purification $\vartheta \in \mathcal{H} \otimes \mathcal{H}^{\prime}$ of $\tau$ with $P(\rho, \tau)=P(\varphi, \vartheta)$.

Proof. We use Uhlmann's theorem to choose $\vartheta \in \mathcal{H} \otimes$ $\mathcal{H}^{\prime}$ such that $F(\rho, \tau)=F(\varphi, \vartheta)$ and, thus, $P(\rho, \tau)=$ $P(\varphi, \vartheta)$.

Corollary 9. Let $\rho, \tau \in \mathcal{S}_{\leq}(\mathcal{H})$ and $\bar{\rho} \in \mathcal{S}_{\leq}\left(\mathcal{H} \otimes \mathcal{H}^{\prime}\right)$ be an extension of $\rho$. Then, there exists an extension $\bar{\tau} \in \mathcal{S}_{\leq}\left(\mathcal{H} \otimes \mathcal{H}^{\prime}\right)$ of $\tau$ with $P(\rho, \tau)=P(\bar{\rho}, \bar{\tau})$.

Proof. Let $\mathcal{H}^{\prime \prime} \cong \mathcal{H} \otimes \mathcal{H}^{\prime}$ be an auxiliary Hilbert space and $\varphi \in \mathcal{H} \otimes \mathcal{H}^{\prime} \otimes \mathcal{H}^{\prime \prime}$ be a purification of $\bar{\rho}$. We introduce a purification $\vartheta \in \mathcal{H} \otimes \mathcal{H}^{\prime} \otimes \mathcal{H}^{\prime \prime}$ of $\tau$ with $P(\varphi, \vartheta)=$ $P(\rho, \tau)$ using Lemma 8 and $\bar{\tau}=\operatorname{tr}_{\mathcal{H}}$ " $\vartheta$. However, due to Lemma 7, we have $P(\varphi, \vartheta) \geq P(\bar{\rho}, \bar{\tau}) \geq P(\rho, \tau)$, which implies that all three distances must be equal.

\section{THE $\varepsilon$-NEIGHBORHOOD INDUCED BY $P$}

The $\varepsilon$-smooth min-entropy of a state $\rho$ is usually defined as a maximization of the min-entropy over a set of states that are $\varepsilon$-close to $\rho$. Various definitions of such sets — subsequently called $\varepsilon$-balls — have appeared 
in the literature. None of the existhing definitions exhibit the following two properties that are of particular importance in the context of smooth conditional minand max-entropies: Firstly, the smooth entropies should be independent of the Hilbert spaces used to represent the state. In particular, embedding the density operator into a larger Hilbert space should leave the smooth entropies unchanged. This can be achieved by allowing sub-normalized states in the $\varepsilon$-balls. Secondly, it will be important that we can define a ball of pure states that contains purifications of all the states in the $\varepsilon$-ball. This allows us to establish the duality relation between smooth min- and max-entropies and is achieved by using a fidelity-based metric to determine $\varepsilon$-closeness. The following ball possesses both of the above properties:

Definition 10. Let $\varepsilon \geq 0$ and $\rho \in \mathcal{S}_{\leq}(\mathcal{H})$ with $\sqrt{\operatorname{tr} \rho}>\varepsilon$. Then, we define an $\varepsilon$-ball in $\mathcal{H}$ around $\rho$ as

$$
\mathcal{B}^{\varepsilon}(\mathcal{H} ; \rho):=\left\{\tau \in \mathcal{S}_{\leq}(\mathcal{H}): P(\tau, \rho) \leq \varepsilon\right\}
$$

We also define $\mathcal{B}_{\mathrm{p}}^{\varepsilon}(\mathcal{H} ; \rho):=\left\{\tau \in \mathcal{B}^{\varepsilon}(\mathcal{H} ; \rho): \operatorname{rank} \tau=1\right\}$, i.e. an $\varepsilon$-ball of pure states around $\rho$.

We now prove some properties of the $\varepsilon$-ball that will be important for our later discussion of smooth conditional min- and max-entropies. Properties i)-iv) clarify what we mean by an $\varepsilon$-ball around $\rho$. Property v) ensures that states in the ball remain in the ball after applying an isometry, while Properties vi) and vii) relate to how the $\varepsilon$-balls change under partial trace and purification. These will be particularly relevant for the duality relation between the smooth min- and max-entropies.

i) The set $\mathcal{B}^{\varepsilon}(\mathcal{H} ; \rho)$ is compact and convex.

Proof. The set is closed and bounded, hence compact. For convexity, we require that, for any $\lambda \in[0,1]$ and $\sigma, \tau \in \mathcal{B}^{\varepsilon}(\mathcal{H} ; \rho)$, the state $\omega:=\lambda \sigma+(1-\lambda) \tau$ is also in $\mathcal{B}^{\varepsilon}(\mathcal{H} ; \rho)$. We define $\hat{\omega}=\omega \oplus(1-\operatorname{tr} \omega)$ and analogously $\hat{\rho}, \hat{\sigma}$ and $\hat{\tau}$. By assumption we have $F(\hat{\sigma}, \hat{\rho}) \geq \sqrt{1-\varepsilon^{2}}$ and $F(\hat{\tau}, \hat{\rho}) \geq \sqrt{1-\varepsilon^{2}}$. We use the concavity of the fidelity (cf. [12], Section 9.2.2) to find

$$
\begin{aligned}
P(\omega, \rho) & =\sqrt{1-F(\hat{\omega}, \hat{\rho})^{2}} \\
& =\sqrt{1-F(\lambda \hat{\sigma}+(1-\lambda) \hat{\tau}, \hat{\rho})^{2}} \\
& \leq \sqrt{1-(\lambda F(\hat{\sigma}, \hat{\rho})+(1-\lambda) F(\hat{\tau}, \hat{\rho}))^{2}} \leq \varepsilon .
\end{aligned}
$$

Therefore, $\omega \in \mathcal{B}^{\varepsilon}(\mathcal{H} ; \rho)$, as required.

ii) Normalized states in $\mathcal{B}^{\varepsilon}(\mathcal{H} ; \rho)$ are not distinguishable from $\rho$ with probability more than $\frac{1}{2}(1+\varepsilon)$.

Proof. By Lemma 6, $\tau \in \mathcal{B}^{\varepsilon}(\mathcal{H} ; \rho)$ implies $\bar{D}(\tau, \rho) \leq$ $P(\tau, \rho) \leq \varepsilon$. The statement then follows from (1).

iii) The ball grows monotonically in the smoothing parameter $\varepsilon$. Furthermore, $\mathcal{B}^{0}(\mathcal{H} ; \rho)=\{\rho\}$. iv) The $\varepsilon$-balls are symmetric and satisfy a triangle inequality. In other words, we have

$$
\begin{aligned}
& \tau \in \mathcal{B}^{\varepsilon}(\mathcal{H} ; \rho) \Longleftrightarrow \rho \in \mathcal{B}^{\varepsilon}(\mathcal{H} ; \tau) \text { and } \\
& \tau \in \mathcal{B}^{\varepsilon}(\mathcal{H} ; \rho) \wedge \sigma \in \mathcal{B}^{\varepsilon^{\prime}}(\mathcal{H} ; \tau) \Longrightarrow \sigma \in \mathcal{B}^{\varepsilon+\varepsilon^{\prime}}(\mathcal{H} ; \rho) .
\end{aligned}
$$

Proof. These properties follow directly from the fact that $P$ is a metric (cf. Lemma 5).

v) The $\varepsilon$-balls are invariant under isometries. Let $U$ : $\mathcal{H} \rightarrow \mathcal{H}^{\prime}$ be an isometry, then

$$
\tau \in \mathcal{B}^{\varepsilon}(\mathcal{H} ; \rho) \Longrightarrow U \tau U^{\dagger} \in \mathcal{B}^{\varepsilon}\left(\mathcal{H}^{\prime} ; U \rho U^{\dagger}\right)
$$

Conversely, if $\Pi$ is the projector onto the image of $U$, then

$$
\sigma \in \mathcal{B}^{\varepsilon}\left(\mathcal{H}^{\prime} ; U \rho U^{\dagger}\right) \Longrightarrow U^{\dagger} \Pi \sigma \Pi U \in \mathcal{B}^{\varepsilon}(\mathcal{H} ; \rho)
$$

Proof. This property follows from Lemma 7 and the fact that $\rho \mapsto U \rho U^{\dagger}$ and $\rho \mapsto U^{\dagger} \Pi \rho \Pi U$ are trace nonincreasing CPMs. ${ }^{3}$

vi) The $\varepsilon$-balls are monotone under partial trace. More precisely, let $\mathcal{H}^{\prime}$ be a Hilbert space and $\operatorname{tr}_{\mathcal{H}}$, the partial trace over $\mathcal{H}^{\prime}$, then

$\tau \in \mathcal{B}^{\varepsilon}\left(\mathcal{H} \otimes \mathcal{H}^{\prime} ; \rho\right) \Longrightarrow \operatorname{tr}_{\mathcal{H}}, \tau \in \mathcal{B}^{\varepsilon}\left(\mathcal{H} ; \operatorname{tr}_{\mathcal{H}}, \rho\right)$

Proof. This property is a direct consequence of Lemma 7 and the fact that the partial trace is a TP-CPM.

vii) On a sufficiently large Hilbert space, there exists a purification of the $\varepsilon$-ball in the following sense: Let $\mathcal{H}^{\prime}$ be a Hilbert space with $\operatorname{dim} \mathcal{H}^{\prime} \geq \operatorname{dim} \mathcal{H}$ and $\varphi \in \mathcal{H} \otimes \mathcal{H}^{\prime}$, then

$$
\begin{aligned}
\tau \in & \mathcal{B}^{\varepsilon}\left(\mathcal{H} ; \operatorname{tr}_{\mathcal{H}^{\prime}} \varphi\right) \Longrightarrow \\
& \exists \vartheta \in \mathcal{B}_{\mathrm{p}}^{\varepsilon}\left(\mathcal{H} \otimes \mathcal{H}^{\prime} ; \varphi\right) \text { s.t. } \tau=\operatorname{tr}_{\mathcal{H}}, \vartheta
\end{aligned}
$$

Proof. This property follows from Lemma $8{ }^{4}$

\footnotetext{
${ }^{3}$ Note that the state $U^{\dagger} \Pi \sigma \Pi U$ is not necessarily normalized. Definitions of $\varepsilon$-balls that do not allow sub-normalized states will not be invariant under isometries in the sense proposed here. This property will be used to show that the smooth min- and max-entropies are invariant under local isometries in Lemmas 13 and 15

4 This property of the $\varepsilon$-ball is due to our use of a fidelity-based metric. In particular, it does not hold for an $\varepsilon$-ball based on the trace distance, such as $\left\{\tau \in \mathcal{S}_{\leq}(\mathcal{H}): \bar{D}(\rho, \tau) \leq \varepsilon\right\}$. We will use this in Lemma 17 to show that the duality relation for the smooth entropy holds.
} 


\section{SMOOTH CONDITIONAL MIN- AND MAX-ENTROPIES}

In this section we define smooth min- and maxentropies and discuss some of their properties that follow from our definition of the $\varepsilon$-ball. In particular, the smooth entropies defined in the following can be seen as optimizations of the corresponding non-smooth entropies over an $\varepsilon$-ball of states (Definition 12 and Lemma [16). Moreover, they are invariant under local isometries (Lemmas 13 and 15) and satisfy a duality relation (Definition 14..$^{5}$

In the following, we assume that $\varepsilon$ is much smaller than the trace of all involved states as is predominantly the case in applications. Indices are used to denote multipartite Hilbert spaces, e.g. $\mathcal{H}_{\mathrm{AB}}=\mathcal{H}_{\mathrm{A}} \otimes \mathcal{H}_{\mathrm{B}}$ and to denote the different marginal states of multi-partite systems. We often do not mention explicitly when a partial trace needs to be taken, e.g. if $\rho_{\mathrm{AB}} \in \mathcal{S}_{\leq}\left(\mathcal{H}_{\mathrm{AB}}\right)$ is given, then $\rho_{\mathrm{A}}:=$ $\operatorname{tr}_{\mathrm{B}} \rho_{\mathrm{AB}}$ is also implicitly defined.

We define the min-entropy:

Definition 11. Let $\rho_{\mathrm{AB}} \in \mathcal{S}_{\leq}\left(\mathcal{H}_{\mathrm{AB}}\right)$, then the minentropy of $\mathrm{A}$ conditioned on $\mathrm{B}$ of $\rho_{\mathrm{AB}}$ is defined as

$H_{\min }(\mathrm{A} \mid \mathrm{B})_{\rho}:=\max _{\sigma_{\mathrm{B}} \in \mathcal{S}_{=}\left(\mathcal{H}_{\mathrm{B}}\right)} \sup \left\{\lambda \in \mathbb{R}: 2^{-\lambda} \mathbb{1}_{\mathrm{A}} \otimes \sigma_{\mathrm{B}} \geq \rho_{\mathrm{AB}}\right\}$.

We now use the $\varepsilon$-ball $\mathcal{B}^{\varepsilon}(\mathcal{H} ; \rho)$ to define a smoothed version of the min-entropy ${ }^{6}$ :

Definition 12. Let $\varepsilon \geq 0$ and $\rho_{\mathrm{AB}} \in \mathcal{S}_{\leq}\left(\mathcal{H}_{\mathrm{AB}}\right)$, then the $\varepsilon$-smooth min-entropy of A conditioned on $\mathrm{B}$ of $\rho_{\mathrm{AB}}$ is defined as

$$
H_{\min }^{\varepsilon}(\mathrm{A} \mid \mathrm{B})_{\rho}:=\max _{\tilde{\rho}_{\mathrm{AB}} \in \mathcal{B}^{\varepsilon}\left(\rho_{\mathrm{AB}}\right)} H_{\min }(\mathrm{A} \mid \mathrm{B})_{\tilde{\rho}}
$$

The quantity is monotonically increasing in $\varepsilon$ due to Property iii) in Section [III and we recover the nonsmooth entropy by $H_{\min }^{0}(\mathrm{~A} \mid \mathrm{B})_{\rho}=H_{\min }(\mathrm{A} \mid \mathrm{B})_{\rho}$. Continuity of the smooth min-entropy as a function of the state is shown in Appendix A

The smooth min-entropy is independent of the Hilbert spaces used to represent the density operator locally, as the following lemma shows:

Lemma 13. Let $\varepsilon \geq 0, \rho_{\mathrm{AB}} \in \mathcal{S}_{\leq}\left(\mathcal{H}_{\mathrm{AB}}\right)$ and $U: \mathcal{H}_{\mathrm{A}} \rightarrow$ $\mathcal{H}_{\mathrm{C}}$ and $V: \mathcal{H}_{\mathrm{B}} \rightarrow \mathcal{H}_{\mathrm{D}}$ be two isometries with $\tau_{\mathrm{CD}}:=$ $(U \otimes V) \rho_{\mathrm{AB}}\left(U^{\dagger} \otimes V^{\dagger}\right)$, then

$$
H_{\min }^{\varepsilon}(\mathrm{A} \mid \mathrm{B})_{\rho}=H_{\min }^{\varepsilon}(\mathrm{C} \mid \mathrm{D})_{\tau} .
$$

\footnotetext{
${ }^{5}$ For convenience of exposition, we will define the smooth maxentropy as the dual of the smooth min-entropy (Definition 14) and then prove that this definition is equivalent to an optimization over an $\varepsilon$-ball of states of the non-smooth max-entropy (Lemma 16).

${ }^{6}$ Note that we drop $\mathcal{H}$ when the Hilbert space is clear from the indices of the state.
}

Proof. First note that the $\varepsilon$-smooth min-entropy $H_{\min }^{\varepsilon}(\mathrm{A} \mid \mathrm{B})$ can be written as

$$
H_{\min }^{\varepsilon}(\mathrm{A} \mid \mathrm{B})_{\rho}=\max _{\tilde{\rho}_{\mathrm{AB}} \in \mathcal{B}^{\varepsilon}\left(\rho_{\mathrm{AB}}\right)} \max _{\substack{\sigma_{\mathrm{B}} \in \mathcal{P}\left(\mathcal{H}_{\mathrm{B}}\right) \\ \tilde{\rho}_{\mathrm{AB}} \leq \mathbb{1}_{\mathrm{A}} \otimes \sigma_{\mathrm{B}}}}-\log \operatorname{tr} \sigma_{\mathrm{B}},
$$

where log denotes the binary logarithm. Now, we let $\bar{\rho}_{\mathrm{AB}} \in \mathcal{B}^{\varepsilon}\left(\rho_{\mathrm{AB}}\right)$ and $\bar{\sigma}_{\mathrm{B}} \in \mathcal{P}\left(\mathcal{H}_{\mathrm{B}}\right)$ be the pair of states that maximize this expression, i.e. $H_{\min }^{\varepsilon}(\mathrm{A} \mid \mathrm{B})_{\rho}=-\log \operatorname{tr} \bar{\sigma}_{\mathrm{B}}$. Then $\bar{\rho}_{\mathrm{AB}} \leq \mathbb{1}_{\mathrm{A}} \otimes \bar{\sigma}_{\mathrm{B}}$ implies

$\underbrace{(U \otimes V) \bar{\rho}_{\mathrm{AB}}\left(U^{\dagger} \otimes V^{\dagger}\right)}_{=: \bar{\tau}_{\mathrm{CD}}} \leq U U^{\dagger} \otimes V \bar{\sigma}_{\mathrm{B}} V^{\dagger} \leq \mathbb{1}_{\mathrm{C}} \otimes \underbrace{V \bar{\sigma}_{\mathrm{B}} V^{\dagger}}_{=: \bar{\omega}_{\mathrm{D}}}$.

The $\varepsilon$-ball is invariant under isometries (cf. Property v)) and, therefore, the pair $\bar{\tau}_{\mathrm{CD}} \in \mathcal{B}^{\varepsilon}\left(\tau_{\mathrm{CD}}\right)$ and $\bar{\omega}_{\mathrm{D}} \in \mathcal{P}\left(\mathcal{H}_{\mathrm{D}}\right)$ is a candidate for the optimization in $H_{\min }^{\varepsilon}(\mathrm{C} \mid \mathrm{D})_{\tau}$. We bound

$$
H_{\min }^{\varepsilon}(\mathrm{C} \mid \mathrm{D})_{\tau} \geq-\log \operatorname{tr} \bar{\omega}_{\mathrm{D}}=-\log \operatorname{tr} \bar{\sigma}_{\mathrm{B}}=H_{\min }^{\varepsilon}(\mathrm{A} \mid \mathrm{B})_{\rho} .
$$

The argument in the reverse direction is similar. Let $\tilde{\tau}_{\mathrm{CD}} \in \mathcal{B}^{\varepsilon}\left(\tau_{\mathrm{CD}}\right)$ and $\tilde{\omega}_{\mathrm{D}} \in \mathcal{P}\left(\mathcal{H}_{\mathrm{D}}\right)$ be the pair that maximizes $H_{\min }^{\varepsilon}(\mathrm{C} \mid \mathrm{D})_{\tau}$. Moreover, we introduce $\Pi_{\mathrm{UV}}=$ $\Pi_{\mathrm{U}} \otimes \Pi_{\mathrm{V}}$, where $\Pi_{\mathrm{U}}$ and $\Pi_{\mathrm{V}}$ are the projectors onto the image of $U$ and $V$ respectively. Then $\tilde{\tau}_{\mathrm{CD}} \leq \mathbb{1}_{\mathrm{C}} \otimes \tilde{\omega}_{\mathrm{D}}$ implies

$$
\underbrace{\left(U^{\dagger} \otimes V^{\dagger}\right) \Pi_{\mathrm{UV}} \tilde{\tau}_{\mathrm{CD}} \Pi_{\mathrm{UV}}(U \otimes V)}_{=: \tilde{\rho}_{\mathrm{AB}}} \leq \mathbb{1}_{\mathrm{A}} \otimes \underbrace{V^{\dagger} \Pi_{\mathrm{V}} \tilde{\omega}_{\mathrm{D}} \Pi_{\mathrm{V}} V}_{=: \tilde{\sigma}_{\mathrm{B}}} .
$$

The pair $\tilde{\rho}_{\mathrm{AB}} \in \mathcal{B}^{\varepsilon}\left(\rho_{\mathrm{AB}}\right)$ and $\tilde{\sigma}_{\mathrm{B}} \in \mathcal{P}\left(\mathcal{H}_{\mathrm{B}}\right)$ is a candidate for the optimization in $H_{\text {min }}^{\varepsilon}(\mathrm{A} \mid \mathrm{B})_{\rho}$ (cf. Property v)) and we get

$$
\begin{aligned}
H_{\min }^{\varepsilon}(\mathrm{A} \mid \mathrm{B})_{\rho} & \geq-\log \operatorname{tr} \tilde{\sigma}_{\mathrm{B}}=-\log \operatorname{tr}\left(\Pi_{\mathrm{V}} \tilde{\omega}_{\mathrm{D}}\right) \\
& \geq-\log \operatorname{tr} \tilde{\omega}_{\mathrm{D}}=H_{\min }^{\varepsilon}(\mathrm{C} \mid \mathrm{D})_{\tau} .
\end{aligned}
$$

We thus conclude that $H_{\min }^{\varepsilon}(\mathrm{A} \mid \mathrm{B})_{\rho}=H_{\min }^{\varepsilon}(\mathrm{C} \mid \mathrm{D})_{\tau}$.

We next define the dual of the smooth min-entropy, the smooth max-entropy:

Definition 14. Let $\varepsilon \geq 0, \rho_{\mathrm{AB}} \in \mathcal{S}_{\leq}\left(\mathcal{H}_{\mathrm{AB}}\right)$ and $\rho_{\mathrm{ABC}} \in$ $\mathcal{S}_{\leq}\left(\mathcal{H}_{\mathrm{ABC}}\right)$ an arbitrary purification of $\rho_{\mathrm{AB}}$, then the $\varepsilon$ smooth max-entropy of A conditioned on $\mathrm{B}$ of $\rho_{\mathrm{AB}}$ is defined as

$$
H_{\max }^{\varepsilon}(\mathrm{A} \mid \mathrm{B})_{\rho}:=-H_{\min }^{\varepsilon}(\mathrm{A} \mid \mathrm{C})_{\rho} .
$$

The quantity is well-defined since all purifications of $\rho_{\mathrm{AB}}$ are equivalent up to an isometry on the purifying space $\mathcal{H}_{\mathrm{C}}$, which does not change $H_{\text {min }}^{\varepsilon}(\mathrm{A} \mid \mathrm{C})$ as Lemma 13 shows. The non-smooth max-entropy is given by $H_{\max }(\mathrm{A} \mid \mathrm{B}):=H_{\max }^{0}(\mathrm{~A} \mid \mathrm{B})$. An alternative expression for the max-entropy was given in [7]:

$$
H_{\max }(\mathrm{A} \mid \mathrm{B})_{\rho}:=\max _{\sigma_{\mathrm{B}} \in \mathcal{S}_{=}\left(\mathcal{H}_{\mathrm{B}}\right)} \log F\left(\rho_{\mathrm{AB}}, \mathbb{1}_{\mathrm{A}} \otimes \sigma_{\mathrm{B}}\right)^{2} .
$$

The smooth max-entropy is independent of the Hilbert spaces used to represent the density operator locally: 
Lemma 15. Let $\varepsilon \geq 0, \rho_{\mathrm{AB}} \in \mathcal{S}_{\leq}\left(\mathcal{H}_{\mathrm{AB}}\right)$, and $U: \mathcal{H}_{\mathrm{A}} \rightarrow$ $\mathcal{H}_{\mathrm{C}}$ and $V: \mathcal{H}_{\mathrm{B}} \rightarrow \mathcal{H}_{\mathrm{D}}$ be two isometries with $\tau_{\mathrm{CD}}:=$ $(U \otimes V) \rho_{\mathrm{AB}}\left(U^{\dagger} \otimes V^{\dagger}\right)$, then

$$
H_{\max }^{\varepsilon}(\mathrm{A} \mid \mathrm{B})_{\rho}=H_{\max }^{\varepsilon}(\mathrm{C} \mid \mathrm{D})_{\tau} .
$$

Proof. Let $\rho_{\mathrm{ABE}}$ be a purification of $\rho_{\mathrm{AB}}$, then $\tau_{\mathrm{CDE}}=$ $\left(U \otimes V \otimes \mathbb{1}_{\mathrm{E}}\right) \rho_{\mathrm{ABE}}\left(U^{\dagger} \otimes V^{\dagger} \otimes \mathbb{1}_{\mathrm{E}}\right)$ is a purification of $\tau_{\mathrm{CD}}$. Thus,

$$
\begin{aligned}
H_{\max }^{\varepsilon}(\mathrm{A} \mid \mathrm{B})_{\rho} & =-H_{\min }^{\varepsilon}(\mathrm{A} \mid \mathrm{E})_{\rho} \\
& =-H_{\min }^{\varepsilon}(\mathrm{C} \mid \mathrm{E})_{\tau}=H_{\max }^{\varepsilon}(\mathrm{C} \mid \mathrm{D})_{\tau} .
\end{aligned}
$$

The $\varepsilon$-smooth max-entropy can also be written as an optimization over an $\varepsilon$-ball of states:

Lemma 16. Let $\varepsilon \geq 0$ and $\rho_{\mathrm{AB}} \in \mathcal{S}_{\leq}\left(\mathcal{H}_{\mathrm{AB}}\right)$, then

$$
H_{\max }^{\varepsilon}(\mathrm{A} \mid \mathrm{B})_{\rho}=\min _{\tilde{\rho}_{\mathrm{AB}} \in \mathcal{B}^{\varepsilon}\left(\rho_{\mathrm{AB}}\right)} H_{\max }(\mathrm{A} \mid \mathrm{B})_{\tilde{\rho}} .
$$

In order to prove the above lemma, we characterize the $\varepsilon$-ball in terms of an $\varepsilon$-ball on the purified space. The following lemma follows directly from Properties vi) and vii) in Section $\amalg$ and will be used repeatedly:

Lemma 17. Let $\rho \in \mathcal{S}_{\leq}(\mathcal{H})$ and $\phi \in \mathcal{S}_{\leq}\left(\mathcal{H} \otimes \mathcal{H}^{\prime}\right)$ be a purification of $\rho$, then

$\mathcal{B}^{\varepsilon}(\mathcal{H} ; \rho) \supseteq\left\{\tilde{\rho} \in \mathcal{S}_{\leq}(\mathcal{H}): \exists \tilde{\phi} \in \mathcal{B}_{\mathrm{p}}^{\varepsilon}\left(\mathcal{H} \otimes \mathcal{H}^{\prime} ; \phi\right)\right.$ s.t. $\left.\tilde{\rho}=\operatorname{tr}_{\mathcal{H}}, \tilde{\phi}\right\}$

and the two sets are identical if the Hilbert space dimensions satisfy $\operatorname{dim} \mathcal{H}^{\prime} \geq \operatorname{dim} \mathcal{H}$.

Proof of Lemma 16, Let $\rho_{\mathrm{ABC}} \in \mathcal{S}_{\leq}\left(\mathcal{H}_{\mathrm{ABC}}\right)$ be a purification of $\rho_{\mathrm{AB}}$ with $\operatorname{dim} \mathcal{H}_{\mathrm{C}} \geq \operatorname{dim} \mathcal{H}_{\mathrm{AB}}$. Then, using Lemma 17 as well as Definition 14 twice each, we have

$$
\begin{aligned}
H_{\max }^{\varepsilon}(\mathrm{A} \mid \mathrm{B})_{\rho} & =-\max _{\tilde{\rho}_{\mathrm{AC}} \in \mathcal{B}^{\varepsilon}\left(\rho_{\mathrm{AC}}\right)} H_{\min }(\mathrm{A} \mid \mathrm{C})_{\tilde{\rho}} \\
& \leq \min _{\tilde{\rho}_{\mathrm{ABC}} \in \mathcal{B}_{\mathrm{p}}^{\varepsilon}\left(\rho_{\mathrm{ABC}}\right)}-H_{\min }(\mathrm{A} \mid \mathrm{C})_{\tilde{\rho}} \\
& =\min _{\tilde{\rho}_{\mathrm{AB}} \in \mathcal{B}^{\varepsilon}\left(\rho_{\mathrm{AB}}\right)} H_{\max }(\mathrm{A} \mid \mathrm{B})_{\tilde{\rho}} .
\end{aligned}
$$

To show the other direction, we choose a purification $\rho_{\mathrm{ABC}} \in \mathcal{S}_{\leq}\left(\mathcal{H}_{\mathrm{ABC}}\right)$ and embed $\mathcal{H}_{\mathrm{B}}$ into a larger space $\mathcal{H}_{\mathrm{B}^{\prime}}$ such that $\operatorname{dim} \mathcal{H}_{\mathrm{B}^{\prime}} \geq \operatorname{dim} \mathcal{H}_{\mathrm{AC}}$. We define $\rho_{\mathrm{AB}^{\prime} \mathrm{C}}$ as the embedding of $\rho_{\mathrm{ABC}}$ into $\mathcal{H}_{\mathrm{AB}^{\prime} \mathrm{C}}$. Furthermore, for each $\tilde{\rho}_{\mathrm{AB}^{\prime} \mathrm{C}} \in \mathcal{B}_{\mathrm{p}}^{\varepsilon}\left(\rho_{\mathrm{AB}^{\prime} \mathrm{C}}\right)$ we construct $\bar{\rho}_{\mathrm{AB}^{\prime} \mathrm{C}}=$ $\left(\mathbb{1}_{\mathrm{A}} \otimes \Pi_{\mathrm{B}} \otimes \mathbb{1}_{\mathrm{C}}\right) \tilde{\rho}_{\mathrm{AB}^{\prime} \mathrm{C}}\left(\mathbb{1}_{\mathrm{A}} \otimes \Pi_{\mathrm{B}} \otimes \mathbb{1}_{\mathrm{C}}\right)$, where $\Pi_{\mathrm{B}}$ is the projector onto $\mathcal{H}_{\mathrm{B}}$, such that $\bar{\rho}_{\mathrm{AB}^{\prime} \mathrm{C}}$ remains in $\mathcal{B}_{\mathrm{p}}^{\varepsilon}\left(\rho_{\mathrm{AB}^{\prime} \mathrm{C}}\right)$ and has support on $\mathcal{H}_{\mathrm{ABC}}$. The set $\left\{\Pi_{\mathrm{B}}, \mathbb{1}_{\mathrm{B}^{\prime}}-\Pi_{\mathrm{B}}\right\}$ describes a measurement on $\mathcal{H}_{\mathrm{B}^{\prime}}$ and the post-measurement state $\bar{\rho}_{\mathrm{AC}} \in \mathcal{S}_{\leq}\left(\mathcal{H}_{\mathrm{AC}}\right)$ satisfies $\bar{\rho}_{\mathrm{AC}} \leq \tilde{\rho}_{\mathrm{AC}}$. Hence, $H_{\min }(\mathrm{A} \mid \mathrm{C})_{\tilde{\rho}} \leq H_{\min }(\mathrm{A} \mid \mathrm{C})_{\bar{\rho}}$.
We may now write (again using Lemma 17 as well as Definition 14 twice each):

$$
\begin{aligned}
H_{\max }^{\varepsilon}(\mathrm{A} \mid \mathrm{B})_{\rho} & =-\max _{\tilde{\rho}_{\mathrm{AC}} \in \mathcal{B}^{\varepsilon}\left(\rho_{\mathrm{AC}}\right)} H_{\min }(\mathrm{A} \mid \mathrm{C})_{\tilde{\rho}} \\
& =\min _{\tilde{\rho}_{\mathrm{AB}^{\prime} \mathrm{C}} \in \mathcal{B}_{\mathrm{p}}^{\varepsilon}\left(\rho_{\mathrm{AB}^{\prime} \mathrm{C}}\right)}-H_{\min }(\mathrm{A} \mid \mathrm{C})_{\tilde{\rho}} \\
& \geq \min _{\bar{\rho}_{\mathrm{ABC}} \in \mathcal{B}_{\mathrm{p}}^{\varepsilon}\left(\rho_{\mathrm{ABC}}\right)}-H_{\min }(\mathrm{A} \mid \mathrm{C})_{\bar{\rho}} \\
& \geq \min _{\bar{\rho}_{\mathrm{AB}} \in \mathcal{B}^{\varepsilon}\left(\rho_{\mathrm{AB}}\right)} H_{\max }(\mathrm{A} \mid \mathrm{B})_{\bar{\rho}},
\end{aligned}
$$

which concludes the proof.

\section{DATA-PROCESSING INEQUALITIES}

As an example of an application of the duality between smooth conditional min- and max-entropies, we consider data-processing inequalities for the two entropies.

We expect measures of uncertainty about the system A given side information $\mathrm{B}$ to be non-decreasing under local physical operations applied to the B system. Here, we show that this is indeed the case for $H_{\min }^{\varepsilon}(\mathrm{A} \mid \mathrm{B})$ and $H_{\max }^{\varepsilon}(\mathrm{A} \mid \mathrm{B})$. The most general physical operations are modeled by TP-CPMs and we denote by $\mathcal{I}_{\mathrm{A}}$ the identity TP-CPM on $\mathcal{P}\left(\mathcal{H}_{\mathrm{A}}\right)$.

Theorem 18. Let $\varepsilon \geq 0, \rho_{\mathrm{AB}} \in \mathcal{S}_{\leq}\left(\mathcal{H}_{\mathrm{AB}}\right)$ and $\mathcal{E}$ : $\mathcal{P}\left(\mathcal{H}_{\mathrm{B}}\right) \rightarrow \mathcal{P}\left(\mathcal{H}_{\mathrm{D}}\right)$ be a TP-CPM with $\tau_{\mathrm{AD}}:=\left(\mathcal{I}_{\mathrm{A}} \otimes\right.$ $\mathcal{E})\left(\rho_{\mathrm{AB}}\right)$, then

$$
\begin{aligned}
& H_{\min }^{\varepsilon}(\mathrm{A} \mid \mathrm{B})_{\rho} \leq H_{\min }^{\varepsilon}(\mathrm{A} \mid \mathrm{D})_{\tau} \quad \text { and } \\
& H_{\max }^{\varepsilon}(\mathrm{A} \mid \mathrm{B})_{\rho} \leq H_{\max }^{\varepsilon}(\mathrm{A} \mid \mathrm{D})_{\tau} .
\end{aligned}
$$

Proof. For a sufficiently large Hilbert space $\mathcal{H}_{\mathrm{R}}$, the TPCPM $\mathcal{E}$ can be decomposed into an isometry $U: \mathcal{H}_{\mathrm{B}} \rightarrow$ $\mathcal{H}_{\mathrm{DR}}$ followed by a partial trace over $\mathcal{H}_{\mathrm{R}}$ (see e.g. [12]). The invariance of the two quantities under local isometries was established in Lemmas 13 and 15, so it remains to show that the quantities are non-decreasing under partial trace $^{7}$, i.e. the inequalities

$$
\begin{aligned}
& H_{\min }^{\varepsilon}(\mathrm{A} \mid \mathrm{DR})_{\tau} \leq H_{\min }^{\varepsilon}(\mathrm{A} \mid \mathrm{D})_{\tau} \quad \text { and } \\
& H_{\max }^{\varepsilon}(\mathrm{A} \mid \mathrm{DR})_{\tau} \leq H_{\max }^{\varepsilon}(\mathrm{A} \mid \mathrm{D})_{\tau} .
\end{aligned}
$$

We first consider the inequality for the smooth minentropy. Let $\tilde{\tau}_{\mathrm{ADR}} \in \mathcal{B}^{\varepsilon}\left(\tau_{\mathrm{ADR}}\right)$ and $\sigma_{\mathrm{DR}} \in \mathcal{P}\left(\mathcal{H}_{\mathrm{DR}}\right)$ be the pair that optimizes the expression in (3) for $H_{\min }^{\varepsilon}(\mathrm{A} \mid \mathrm{DR})_{\tau}$, then

$$
\tilde{\tau}_{\mathrm{ADR}} \leq \mathbb{1}_{\mathrm{A}} \otimes \sigma_{\mathrm{DR}} \Longrightarrow \tilde{\tau}_{\mathrm{AD}} \leq \mathbb{1}_{\mathrm{A}} \otimes \sigma_{\mathrm{D}}
$$

\footnotetext{
7 This property is sometimes referred to as strong sub-additivity of the smooth min- and max-entropies. This is due to the fact that $H(\mathrm{~A} \mid \mathrm{BC}) \leq H(\mathrm{~A} \mid \mathrm{B})$ is equivalent to the strong sub-additivity of the von Neumann entropy.
} 
and, due to Property vi), the pair $\tilde{\tau}_{\mathrm{AD}} \in \mathcal{B}^{\varepsilon}\left(\tau_{\mathrm{AD}}\right)$ and $\sigma_{\mathrm{D}} \in$ $\mathcal{P}\left(\mathcal{H}_{\mathrm{D}}\right)$ is a candidate for the optimization in $H_{\text {min }}^{\varepsilon}(\mathrm{A} \mid \mathrm{D})_{\tau}$. Thus, $H_{\min }^{\varepsilon}(\mathrm{A} \mid \mathrm{DR})_{\tau} \leq H_{\min }^{\varepsilon}(\mathrm{A} \mid \mathrm{D})_{\tau}$.

For the smooth max-entropy, let $\tau_{\mathrm{ADRE}} \in \mathcal{S}_{\leq}\left(\mathcal{H}_{\mathrm{ADRE}}\right)$ be a purification of $\tau_{\mathrm{ADR}}$, then

$$
\begin{aligned}
H_{\max }^{\varepsilon}(\mathrm{A} \mid \mathrm{DR})_{\tau} & =-H_{\min }^{\varepsilon}(\mathrm{A} \mid \mathrm{E})_{\tau} \\
& \leq-H_{\min }^{\varepsilon}(\mathrm{A} \mid \mathrm{ER})_{\tau}=H_{\max }^{\varepsilon}(\mathrm{A} \mid \mathrm{D})_{\tau}
\end{aligned}
$$

which concludes the proof.

The second pair of data-processing inequalities concerns projective (von Neumann) measurements of the system A. Such measurements can be described in terms of an orthonormal basis $\left\{|i\rangle_{\mathrm{A}}\right\}_{i}$ of $\mathcal{H}_{\mathrm{A}}$ and a TP-CPM $\mathcal{M}$ from $\mathcal{H}_{\mathrm{A}}$ to $\mathcal{H}_{\mathrm{X}} \cong \mathcal{H}_{\mathrm{A}}$ which maps $\rho_{\mathrm{A}}$ to $\sum_{i}\left\langle i\left|\rho_{\mathrm{A}}\right| i\right\rangle|i\rangle\left\langle\left. i\right|_{\mathrm{X}}\right.$. We expect that the uncertainty about the system $\mathrm{A}$ as well as the entropies $H_{\min }^{\varepsilon}(\mathrm{AB} \mid \mathrm{C})$ and $H_{\max }^{\varepsilon}(\mathrm{AB} \mid \mathrm{C})$ will not decrease with such a measurement.

Theorem 19. Let $\varepsilon \geq 0, \rho_{\mathrm{ABC}} \in \mathcal{S}_{\leq}\left(\mathcal{H}_{\mathrm{ABC}}\right)$ and $\mathcal{M}$ : $\mathcal{P}\left(\mathcal{H}_{\mathrm{A}}\right) \rightarrow \mathcal{P}\left(\mathcal{H}_{\mathrm{X}}\right)$ a TP-CPM describing a projective measurement with $\tau_{\mathrm{XBC}}:=\left(\mathcal{M} \otimes \mathcal{I}_{\mathrm{BC}}\right)\left(\rho_{\mathrm{ABC}}\right)$. Then,

$$
\begin{aligned}
& H_{\min }^{\varepsilon}(\mathrm{AB} \mid \mathrm{C})_{\rho} \leq H_{\min }^{\varepsilon}(\mathrm{XB} \mid \mathrm{C})_{\tau} \quad \text { and } \\
& H_{\max }^{\varepsilon}(\mathrm{AB} \mid \mathrm{C})_{\rho} \leq H_{\max }^{\varepsilon}(\mathrm{XB} \mid \mathrm{C})_{\tau} .
\end{aligned}
$$

Proof. Note that $\mathcal{M}$ can be decomposed into an isometry $U: \mathcal{H}_{\mathrm{A}} \rightarrow \mathcal{H}_{\mathrm{x}} \otimes \mathcal{H}_{\mathrm{x}}^{\prime}, \mathcal{H}_{\mathrm{x}}^{\prime} \cong \mathcal{H}_{\mathrm{x}}$ that maps $|i\rangle_{\mathrm{A}}$ to $|i\rangle_{\mathrm{X}} \otimes$ $|i\rangle_{\mathrm{X}^{\prime}}$ followed by a partial trace over $\mathcal{H}_{\mathrm{X}}^{\prime}$. We denote the intermediate state by $\tau_{\mathrm{Xx}{ }^{\prime} \mathrm{BC}}$ and the projector onto the image of $U$ by $\Pi_{\mathrm{xx}^{\prime}}$. Moreover, Note that $\mathcal{M}\left(\mathbb{1}_{\mathrm{A}}\right)=\mathbb{1}_{\mathrm{x}}$.

We first prove the statement for the min-entropy. Let $\tilde{\rho}_{\mathrm{ABC}} \in \mathcal{B}^{\varepsilon}\left(\rho_{\mathrm{ABC}}\right)$ and $\sigma_{\mathrm{C}} \in \mathcal{P}\left(\mathcal{H}_{\mathrm{C}}\right)$ such that $H_{\min }^{\varepsilon}(\mathrm{AB} \mid \mathrm{C})_{\rho}=H_{\min }(\mathrm{AB} \mid \mathrm{C})_{\tilde{\rho}}=-\log \operatorname{tr} \sigma_{\mathrm{C}}$. Then, $\tilde{\rho}_{\mathrm{ABC}} \leq \mathbb{1}_{\mathrm{AB}} \otimes \sigma_{\mathrm{C}}$ implies

$$
\left(\mathcal{M} \otimes \mathcal{I}_{\mathrm{BC}}\right)\left(\tilde{\rho}_{\mathrm{ABC}}\right) \leq\left(\mathcal{M} \otimes \mathcal{I}_{\mathrm{BC}}\right)\left(\mathbb{1}_{\mathrm{AB}} \otimes \sigma\right)=\mathbb{1}_{\mathrm{XB}} \otimes \sigma_{\mathrm{C}} .
$$

The state $\tilde{\tau}_{\mathrm{XBC}}:=\left(\mathcal{M} \otimes \mathcal{I}_{\mathrm{BC}}\right)\left(\tilde{\rho}_{\mathrm{ABC}}\right)$ is in $\mathcal{B}^{\varepsilon}\left(\tau_{\mathrm{XBC}}\right)$ due to Lemma 7. Hence, $\tilde{\tau}_{\mathrm{XBC}}$ with $\sigma_{\mathrm{C}}$ is a candidate for the optimization in $H_{\min }^{\varepsilon}(\mathrm{XB} \mid \mathrm{C})_{\tau}$ and, thus, $H_{\min }^{\varepsilon}(\mathrm{XB} \mid \mathrm{C})_{\tau} \geq$ $H_{\min }^{\varepsilon}(\mathrm{AB} \mid \mathrm{C})_{\rho}$.

To prove the statement for the max-entropy, we let $\bar{\tau}_{\mathrm{XBC}} \in \mathcal{B}^{\varepsilon}\left(\tau_{\mathrm{XBC}}\right)$ be such that $H_{\max }^{\varepsilon}(\mathrm{XB} \mid \mathrm{C})_{\tau}=$ $H_{\max }(\mathrm{XB} \mid \mathrm{C})_{\bar{\tau}}$. We use Corollary 9 to introduce its extension $\bar{\tau}_{\mathrm{XX^{ \prime } \mathrm { BC }}} \in \mathcal{B}^{\varepsilon}\left(\tau_{\mathrm{XX}^{\prime} \mathrm{BC}}\right)$. Furthermore, we employ (5) to get

$$
\begin{aligned}
H_{\max }^{\varepsilon}(\mathrm{XB} \mid \mathrm{C})_{\tau} & =\max _{\sigma_{\mathrm{B}} \in \mathcal{S}_{=}\left(\mathcal{H}_{\mathrm{B}}\right)} \log F\left(\bar{\tau}_{\mathrm{XBC}}, \mathbb{1}_{\mathrm{XB}} \otimes \sigma_{\mathrm{C}}\right)^{2} \\
& \geq \max _{\sigma_{\mathrm{B}} \in \mathcal{S}_{=}\left(\mathcal{H}_{\mathrm{B}}\right)} \log F\left(\bar{\tau}_{\mathrm{Xx}^{\prime} \mathrm{BC}}, \Pi_{\mathrm{Xx}^{\prime}} \otimes \mathbb{1}_{\mathrm{B}} \otimes \sigma_{\mathrm{C}}\right)^{2} \\
& =\max _{\sigma_{\mathrm{B}} \in \mathcal{S}_{=}\left(\mathcal{H}_{\mathrm{B}}\right)} \log F\left(\breve{\tau}_{\mathrm{Xx}^{\prime} \mathrm{BC}}, \mathbb{1}_{\mathrm{XX}^{\prime} \mathrm{B}} \otimes \sigma_{\mathrm{C}}\right)^{2} \\
& =H_{\max }\left(\mathrm{XX}^{\prime} \mathrm{B} \mid \mathrm{C}\right)_{\breve{\tau}}
\end{aligned}
$$

where we used that the fidelity can only increase under partial trace and introduced the state $\breve{\tau}_{\mathrm{XX}^{\prime} \mathrm{BC}}:=$ $\left(\Pi_{\mathrm{XX}^{\prime}} \otimes \mathbb{1}_{\mathrm{BC}}\right) \bar{\tau}_{\mathrm{XX}^{\prime} \mathrm{BC}}\left(\Pi_{\mathrm{XX}^{\prime}} \otimes \mathbb{1}_{\mathrm{BC}}\right)$. We have $\breve{\tau}_{\mathrm{XX}^{\prime} \mathrm{BC}} \in$
$\mathcal{B}^{\varepsilon}\left(\tau_{\mathrm{Xx}^{\prime} \mathrm{BC}}\right)$ due to the definition of $\bar{\tau}_{\mathrm{Xx}^{\prime} \mathrm{BC}}$ and Lemma 7 . We use this and Lemma 15 to write $H_{\max }\left(\mathrm{XX}^{\prime} \mathrm{B} \mid \mathrm{C}\right)_{\breve{\tau}} \geq$ $H_{\max }^{\varepsilon}\left(\mathrm{XX}^{\prime} \mathrm{B} \mid \mathrm{C}\right)_{\tau}=H_{\max }^{\varepsilon}(\mathrm{AB} \mid \mathrm{C})_{\rho}$, from which the lemma follows.

Note that, in conjunction with the fully quantum generalization of the AEP (Theorem 1 in [6]), the inequalities in Theorem 18 and 19 imply the same inequalities for the von Neumann entropy.

\section{APPENDIX A: TECHNICAL RESULTS}

Here, we establish some useful properties of the minand max-entropies. In particular, we give bounds on the min- and max-entropies in terms of the Hilbert space dimensions, show their continuity as a function of the state and prove that the max-entropy is concave. Properties analogous to the ones we present here are also found for the von Neumann entropy (see e.g. [12, 16]).

\section{Preliminaries}

Let us consider the functional $\Phi: \rho_{\mathrm{AB}} \mapsto 2^{-H_{\min }(\mathrm{A} \mid \mathrm{B})_{\rho}}$, which we extend to arbitrary Hermitian operators, $\mathcal{L}^{\dagger}\left(\mathcal{H}_{\mathrm{AB}}\right)$, on $\mathcal{H}_{\mathrm{AB}}$ as follows:

$$
\Phi: \mathcal{L}^{\dagger}\left(\mathcal{H}_{\mathrm{AB}}\right) \rightarrow \mathbb{R}, \rho_{\mathrm{AB}} \mapsto \inf _{\substack{\sigma_{\mathrm{B}} \in \mathcal{L}^{\dagger}\left(\mathcal{H}_{\mathrm{B}}\right) \\ \rho_{\mathrm{AB}} \leq \mathbb{1}_{\mathrm{A}} \otimes \sigma_{\mathrm{B}}}} \operatorname{tr} \sigma_{\mathrm{B}} .
$$

The functional has the following properties:

i) Multiplication with scalar: Let $\lambda \geq 0$, then $\Phi\left(\lambda \rho_{\mathrm{AB}}\right)=\lambda \Phi\left(\rho_{\mathrm{AB}}\right)$.

ii) Monotonicity: $\rho_{\mathrm{AB}} \geq \tau_{\mathrm{AB}} \Longrightarrow \Phi\left(\rho_{\mathrm{AB}}\right) \geq \Phi\left(\tau_{\mathrm{AB}}\right)$.

iii) Sub-Additivity: $\Phi\left(\rho_{\mathrm{AB}}+\tau_{\mathrm{AB}}\right) \leq \Phi\left(\rho_{\mathrm{AB}}\right)+\Phi\left(\tau_{\mathrm{AB}}\right)$. Furthermore, equality holds if $\operatorname{tr}\left(\rho_{\mathrm{B}} \tau_{\mathrm{B}}\right)=0$.

iv) Bounds: Let $d_{\mathrm{A}}=\operatorname{dim} \mathcal{H}_{\mathrm{A}}$ and $d_{\text {min }}=$ $\min \left\{d_{\mathrm{A}}, \operatorname{dim} \mathcal{H}_{\mathrm{B}}\right\}$, then $\frac{1}{d_{\mathrm{A}}} \operatorname{tr} \rho_{\mathrm{AB}} \leq \Phi\left(\rho_{\mathrm{AB}}\right) \leq$ $d_{\min } \operatorname{tr}\left\{\rho_{\mathrm{AB}}\right\}_{+}$.

Proof. To get the upper bound, first consider a normalized pure state $\phi_{\mathrm{AB}}$. Clearly, $\phi_{\mathrm{AB}} \leq \Pi_{\mathrm{A}}^{\phi} \otimes \Pi_{\mathrm{B}}^{\phi} \leq \mathbb{1}_{\mathrm{A}} \otimes \Pi_{\mathrm{B}}^{\phi}$, where $\Pi_{\mathrm{A}}^{\phi}$ and $\Pi_{\mathrm{B}}^{\phi}$ are the projectors onto the support of $\phi_{\mathrm{A}}$ and $\phi_{\mathrm{B}}$, respectively. Furthermore $\operatorname{tr} \Pi_{\mathrm{B}}^{\phi} \leq d$ thanks to the Schmidt decomposition. Using the eigenvalue decomposition $\rho_{\mathrm{AB}}=\sum_{i} \lambda_{i} \phi_{\mathrm{AB}}^{i}$, we get

$$
\Phi\left(\rho_{\mathrm{AB}}\right) \leq \operatorname{tr}\left(\sum_{i: \lambda_{i}>0} \lambda_{i} \Pi_{\mathrm{B}}^{\phi^{i}}\right) \leq d_{\min } \operatorname{tr}\left\{\rho_{\mathrm{AB}}\right\}_{+} .
$$

On the other hand, we have $\operatorname{tr}\left(\mathbb{1}_{\mathrm{A}} \otimes \sigma_{\mathrm{B}}\right) \geq \operatorname{tr} \rho_{\mathrm{AB}}$ for any candidate $\sigma_{\mathrm{B}}$, hence,

$$
\Phi\left(\rho_{\mathrm{AB}}\right) \geq \frac{1}{d_{\mathrm{A}}} \operatorname{tr} \rho_{\mathrm{AB}} .
$$


Properties i) and iii) imply convexity of $\Phi$, i.e. $\Phi\left(\lambda \rho_{\mathrm{AB}}+(1-\lambda) \tau_{\mathrm{AB}}\right) \leq \lambda \Phi\left(\rho_{\mathrm{AB}}\right)+(1-\lambda) \Phi\left(\tau_{\mathrm{AB}}\right)$.

\section{Bounds on the Conditional Entropies}

In [6] it was shown that, for $\bar{\rho}_{\mathrm{AB}} \in \mathcal{S}_{=}\left(\mathcal{H}_{\mathrm{AB}}\right)$, we have $H_{\min }(\mathrm{A} \mid \mathrm{B})_{\bar{\rho}} \leq H_{\max }(\mathrm{A} \mid \mathrm{B})_{\bar{\rho}}$. For sub-normalized states $\rho_{\mathrm{AB}}=\operatorname{tr} \rho_{\mathrm{AB}} \cdot \bar{\rho}_{\mathrm{AB}}$, we thus have

$$
H_{\text {min }}(\mathrm{A} \mid \mathrm{B})_{\rho}+\log \operatorname{tr} \rho_{\mathrm{AB}} \leq H_{\max }(\mathrm{A} \mid \mathrm{B})_{\rho}-\log \operatorname{tr} \rho_{\mathrm{AB}} .
$$

We now establish bounds on the min- and maxentropies:

Lemma 20. Let $\rho_{\mathrm{AB}} \in \mathcal{S}_{\leq}\left(\mathcal{H}_{\mathrm{AB}}\right), d_{\mathrm{A}}=\operatorname{dim} \mathcal{H}_{\mathrm{A}}$ and $d_{\text {min }}=\min \left\{d_{\mathrm{A}}, \operatorname{dim} \mathcal{H}_{\mathrm{B}}\right\}$, then

$$
\begin{aligned}
& -\log d_{\min } \leq H_{\min }(\mathrm{A} \mid \mathrm{B})_{\rho}+\log \operatorname{tr} \rho_{\mathrm{AB}} \leq \log d_{\mathrm{A}} \\
& -\log d_{\min } \leq H_{\max }(\mathrm{A} \mid \mathrm{B})_{\rho}-\log \operatorname{tr} \rho_{\mathrm{AB}} \leq \log d_{\mathrm{A}} .
\end{aligned}
$$

Proof. The bounds on the min-entropy follow directly from (A1) and (A2). The bounds on the max-entropy follow by duality (4) and (A3).

\section{Continuity of the Conditional Entropies}

The operational interpretation of the conditional minentropy as a guessing probability (cf. [7]) already implies its continuity in the state. To see this, note that a discontinuity in the guessing probability could be detected experimentally using a fixed number of trials (the number depending only on the required precision), hence giving us the means to distinguish between arbitrarily close states for a cost (in terms of the number of trials) independent of their distance. For sufficiently close states, this would contradict the upper bound on the distinguishing advantage (11). Here, we make this statement more precise.

Lemma 21. Let $\rho_{\mathrm{AB}}, \tau_{\mathrm{AB}} \in \mathcal{S}_{\leq}\left(\mathcal{H}_{\mathrm{AB}}\right)$ and $\delta:=$ $\bar{D}\left(\rho_{\mathrm{AB}}, \tau_{\mathrm{AB}}\right)$, then

$$
\left|H_{\min }(\mathrm{A} \mid \mathrm{B})_{\rho}-H_{\min }(\mathrm{A} \mid \mathrm{B})_{\tau}\right| \leq \frac{d_{\mathrm{A}} d_{\min } \delta}{\ln 2 \cdot \min \left\{\operatorname{tr} \rho_{\mathrm{AB}}, \operatorname{tr} \tau_{\mathrm{AB}}\right\}} .
$$

Proof. We use continuity of the functional $\Phi$ to obtain

$$
\begin{aligned}
\Phi\left(\tau_{\mathrm{AB}}\right) & =\Phi\left(\rho_{\mathrm{AB}}+\left(\tau_{\mathrm{AB}}-\rho_{\mathrm{AB}}\right)\right) \leq \Phi\left(\rho_{\mathrm{AB}}\right)+\Phi\left(\tau_{\mathrm{AB}}-\rho_{\mathrm{AB}}\right) \\
& \leq \Phi\left(\rho_{\mathrm{AB}}\right)+d_{\min } \operatorname{tr}\left\{\tau_{\mathrm{AB}}-\rho_{\mathrm{AB}}\right\}_{+} \leq \Phi\left(\rho_{\mathrm{AB}}\right)+d_{\min } \delta .
\end{aligned}
$$

Note that $\Phi>0$ for all states in $\mathcal{S}_{\leq}\left(\mathcal{H}_{\mathrm{AB}}\right)$. Taking the logarithm and using the bound $\ln (a+x) \leq \ln a+\frac{x}{a}$, we find

$$
\log \Phi\left(\tau_{\mathrm{AB}}\right)-\log \Phi\left(\rho_{\mathrm{AB}}\right) \leq \frac{d_{\min } \delta}{\ln 2 \cdot \Phi\left(\rho_{\mathrm{AB}}\right)} \leq \frac{d_{\mathrm{A}} d_{\min } \delta}{\ln 2 \cdot \operatorname{tr} \rho_{\mathrm{AB}}} .
$$

The same argument also applies on exchange of $\rho_{\mathrm{AB}}$ and $\tau_{\mathrm{AB}}$ and we obtain the statement of the lemma by substituting $H_{\min }(\mathrm{A} \mid \mathrm{B})_{\rho}=-\log \Phi\left(\rho_{\mathrm{AB}}\right)$.
Remark 22. The above result is tight in the following sense: Consider a system with Hilbert spaces $\mathcal{H}_{\mathrm{A}}$ and $\mathcal{H}_{\mathrm{B}}=\mathcal{H}_{\mathrm{A}}^{\prime} \oplus \mathcal{H}_{\mathrm{B}}^{\prime}$, where $\mathcal{H}_{\mathrm{A}}^{\prime} \cong \mathcal{H}_{\mathrm{A}}$. Let $\psi_{\mathrm{AB}}$ be the normalized fully entangled state on $\mathcal{H}_{\mathrm{A}} \otimes \mathcal{H}_{\mathrm{A}}^{\prime}$ and $\rho_{\mathrm{B}} \in \mathcal{S}_{\leq}\left(\mathcal{H}_{\mathrm{B}}^{\prime}\right)$ be orthogonal to $\psi_{\mathrm{B}}$. The choice $\rho_{\mathrm{AB}}=\frac{\mathbb{1}_{\mathrm{A}}}{d_{\mathrm{A}}} \otimes \rho_{\mathrm{B}}$ and $\tau_{\mathrm{AB}}=\rho_{\mathrm{AB}}+\delta \psi_{\mathrm{AB}}$ for some small $\delta>0$ leads to $\bar{D}\left(\rho_{\mathrm{AB}}, \tau_{\mathrm{AB}}\right)=\delta$,

$$
\Phi\left(\rho_{\mathrm{AB}}\right)=\frac{\operatorname{tr} \rho_{\mathrm{B}}}{d_{\mathrm{A}}} \quad \text { and } \quad \Phi\left(\tau_{\mathrm{AB}}\right)=\Phi\left(\rho_{\mathrm{AB}}\right)+d_{\min } \delta .
$$

Taking the logarithm (for small $\delta$ ) leads to

$$
\log \Phi\left(\tau_{\mathrm{AB}}\right)-\log \Phi\left(\rho_{\mathrm{AB}}\right) \approx \frac{d_{\min } \delta}{\ln 2 \cdot \Phi\left(\rho_{\mathrm{AB}}\right)}=\frac{d_{\mathrm{A}} d_{\min } \delta}{\ln 2 \cdot \operatorname{tr} \rho_{\mathrm{AB}}} .
$$

Lemma 21 implies that the conditional min-entropy is uniformly (Lipschitz) continuous on the set of normalized states and in any $\varepsilon$-ball. Since $\bar{D}(\rho, \tau) \leq P(\rho, \tau)$ (cf. Lemma 6i), Lemma 21 also holds for $\delta=P\left(\rho_{\mathrm{AB}}, \tau_{\mathrm{AB}}\right)$.

The continuity of the smooth min- and max-entropies then follows: Let $\tilde{\rho}_{\mathrm{AB}} \in \mathcal{B}^{\varepsilon}\left(\rho_{\mathrm{AB}}\right)$ be such that $H_{\min }^{\varepsilon}(\mathrm{A} \mid \mathrm{B})_{\rho}=H_{\min }(\mathrm{A} \mid \mathrm{B})_{\tilde{\rho}}$. We now construct a state $\tilde{\tau}_{\mathrm{AB}}$ that is $\varepsilon$-close to $\tau_{\mathrm{AB}}$ and $\delta^{\prime}$-close to $\tilde{\rho}_{\mathrm{AB}}$, where $\delta^{\prime}:={\sqrt{\delta^{2}+2 \varepsilon \delta}}^{8}$ We get

$H_{\min }^{\varepsilon}(\mathrm{A} \mid \mathrm{B})_{\rho}-H_{\min }^{\varepsilon}(\mathrm{A} \mid \mathrm{B})_{\tau} \leq H_{\min }(\mathrm{A} \mid \mathrm{B})_{\tilde{\rho}}-H_{\min }(\mathrm{A} \mid \mathrm{B})_{\tilde{\tau}}$,

which vanishes continuously for $\delta \rightarrow 0$ due to Lemma 21. The continuity of the smooth max-entropy follows by duality (4). Using Lemma 7, we introduce purifications $\rho_{\mathrm{ABC}}$ of $\rho_{\mathrm{AB}}$ and $\tau_{\mathrm{ABC}}$ of $\tau_{\mathrm{AB}}$ such that $P\left(\rho_{\mathrm{AB}}, \tau_{\mathrm{AB}}\right)=$ $P\left(\rho_{\mathrm{ABC}}, \tau_{\mathrm{ABC}}\right) \geq P\left(\rho_{\mathrm{AC}}, \tau_{\mathrm{AC}}\right)$. Then,

$\left|H_{\max }^{\varepsilon}(\mathrm{A} \mid \mathrm{B})_{\rho}-H_{\text {max }}^{\varepsilon}(\mathrm{A} \mid \mathrm{B})_{\tau}\right|=\left|H_{\min }^{\varepsilon}(\mathrm{A} \mid \mathrm{C})_{\rho}-H_{\min }^{\varepsilon}(\mathrm{A} \mid \mathrm{C})_{\tau}\right|$

which can be bounded using Lemma 21 with $d_{\min }=d_{\mathrm{A}}$.

\section{Concavity of the Max-Entropy}

The max-entropy is a concave function of the state.

Lemma 23. Let $\left\{p_{i}\right\}_{i}$ be a probability distribution, $\left\{\rho_{\mathrm{AB}}^{i}\right\}_{i}$ be a set of states in $\mathcal{S}_{\leq}\left(\mathcal{H}_{\mathrm{AB}}\right)$ and $\tau_{\mathrm{AB}}:=$ $\sum_{i} p_{i} \rho_{\mathrm{AB}}^{i}$. Then,

$$
H_{\max }(\mathrm{A} \mid \mathrm{B})_{\tau} \geq \sum_{i} p_{i} H_{\max }(\mathrm{A} \mid \mathrm{B})_{\rho^{i}}
$$

\footnotetext{
8 The construction is as follows: Let $c:=(\delta+\varepsilon)^{2}, \varphi$ be a purification of $\tilde{\rho}_{\mathrm{AB}} \oplus\left(1-\operatorname{tr} \tilde{\rho}_{\mathrm{AB}}\right)$ and $\vartheta$ a be purification of $\tau_{\mathrm{AB}} \oplus\left(1-\operatorname{tr} \tau_{\mathrm{AB}}\right)$ such that $|\langle\varphi \mid \vartheta\rangle|^{2}=\bar{F}\left(\tilde{\rho}_{\mathrm{AB}}, \tau_{\mathrm{AB}}\right)^{2} \geq 1-c$. We choose $\tilde{\tau}_{\mathrm{AB}}:=c^{-1}\left(\varepsilon^{2} \tilde{\rho}_{\mathrm{AB}}+\delta^{\prime 2} \tau_{\mathrm{AB}}\right)$. Now, $P\left(\tilde{\tau}_{\mathrm{AB}}, \tau_{\mathrm{AB}}\right)^{2} \leq$ $P\left(c^{-1}\left(\varepsilon^{2} \varphi+\delta^{\prime 2} \vartheta\right), \vartheta\right)^{2}=1-c^{-1}\left\langle\vartheta\left|\varepsilon^{2} \varphi+\delta^{\prime 2} \vartheta\right| \vartheta\right\rangle \leq 1-c^{-1}\left(\delta^{\prime 2}+\right.$ $\left.\varepsilon^{2}(1-c)\right)=\varepsilon^{2}$. Similarly, $P\left(\tilde{\tau}_{\mathrm{AB}}, \tilde{\rho}_{\mathrm{AB}}\right) \leq \delta^{\prime}$.
} 
Proof. Let $\left|\varphi^{i}\right\rangle_{\mathrm{ABC}}$ purify $\rho_{\mathrm{AB}}^{i}$ such that the state $|\tau\rangle_{\mathrm{ABCYZ}}:=\sum_{i} \sqrt{p_{i}}\left|\varphi^{i}\right\rangle_{\mathrm{ABC}} \otimes|i\rangle_{\mathrm{Y}} \otimes|i\rangle_{\mathrm{Z}}$ - where $\left\{|i\rangle_{\mathrm{Y}}\right\}_{i}$ and $\left\{|i\rangle_{\mathrm{z}}\right\}_{i}$ are orthonormal bases of the auxiliary Hilbert spaces $\mathcal{H}_{\mathrm{Y}}$ and $\mathcal{H}_{\mathrm{Z}}$, respectively - has marginals $\tau_{\mathrm{AB}}$ and $\tau_{\mathrm{ACZ}}=\sum_{i} p_{i} \rho_{\mathrm{AC}}^{i} \otimes|i\rangle\left\langle\left. i\right|_{\mathrm{z}}\right.$. Using data-processing of the max-entropy (Theorem [18), the properties of $\Phi$ and the concavity of the logarithm, we find

$$
\begin{aligned}
H_{\max }(\mathrm{A} \mid \mathrm{B})_{\tau} & \geq H_{\max }(\mathrm{A} \mid \mathrm{BY})_{\tau}=-H_{\min }(\mathrm{A} \mid \mathrm{CZ})_{\tau} \\
& =\log \Phi\left(\tau_{\mathrm{A}(\mathrm{CZ})}\right)=\log \left(\sum_{i} p_{i} \Phi\left(\rho_{\mathrm{AC}}^{i}\right)\right) \\
& \geq \sum_{i} p_{i} \log \Phi\left(\rho_{\mathrm{AC}}^{i}\right)=\sum_{i} p_{i} H_{\max }(\mathrm{A} \mid \mathrm{B})_{\rho^{i}} .
\end{aligned}
$$

Note also that the min-entropy is neither a concave nor a convex function of the state.

\section{ACKNOWLEDGMENT}

We thank Nilanjana Datta, Jürg Wullschleger and Christian Schaffner for fruitful discussions and comments. We acknowledge support from the Swiss National Science Foundation (grant No. 200021-119868).
[1] R. Renner and R. König, in Second Theory of Cryptography Conference, TCC 2005 (Springer, 2005), vol. 3378 of $L N C S$, pp. 407-425.

[2] R. Renner, Ph.D. thesis (2005), URL http://arxiv.org/abs/quant-ph/0512258

[3] R. Renner and S. Wolf, in Advances in Cryptology ASIACRYPT (Springer, 2005), vol. 3788 of Lecture Notes in Computer Science, pp. 199-216.

[4] M. Berta, Master's thesis (2008).

[5] F. Buscemi and N. Datta (2009), URL http://arxiv.org/abs/0902.0158v5

[6] M. Tomamichel, R. Colbeck, and R. Renner (2008), URL http://arxiv.org/abs/0811.1221v3.

[7] R. König, R. Renner, and C. Schaffner, IEEE Trans. on Inf. Theory 55, 4337 (2009), URL http://arxiv.org/abs/0807.1338.

[8] T. S. Han, Information-Spectrum Methods in Information Theory (Springer, 2002).

[9] M. Hayashi and H. Nagaoka, IEEE Trans. on Inf. Theory
49, 1753 (2002).

[10] H. Nagaoka and M. Hayashi, IEEE Trans. on Inf. Theory 53, 534 (2007).

[11] N. Datta and R. Renner, Smooth rényi entropies and the quantum information spectrum (2008), URL http://arxiv.org/abs/0801.0282

[12] M. A. Nielsen and I. L. Chuang, Quantum computation and quantum information (Cambridge University Press, 2000).

[13] A. Uhlmann, Annalen der Physik 497, 524 (1985).

[14] A. Gilchrist, N. K. Langford, and M. A. Nielsen, Distance measures to compare real and ideal quantum processes (2004), URL http://arxiv.org/abs/quant-ph/0408063

[15] A. E. Rastegin, Sine distance for quantum states (2006), URL http://arxiv.org/abs/quant-ph/0602112v1.

[16] R. Alicki and M. Fannes (2003), URL http://arxiv.org/abs/quant-ph/0312081v1 